\title{
Radiomics Study of Thyroid Ultrasound for Predicting BRAF Mutation in Papillary Thyroid Carcinoma: Preliminary Results
}

\author{
(D) M.-r. Kwon, (D).H. Shin, (D) H. Park, (D) H. Cho, (D) S.Y. Hahn, and (D) K.W. Park
}

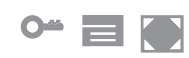

\begin{abstract}
BACKGROUND AND PURPOSE: It is not known how radiomics using ultrasound images contribute to the detection of BRAF mutation. This study aimed to evaluate whether a radiomics study of gray-scale ultrasound can predict the presence or absence of $B$ Raf proto-oncogene, serine/threonine kinase (BRAF) mutation in papillary thyroid cancer.
\end{abstract}

\begin{abstract}
MATERIALS AND METHODS: The study retrospectively included 96 thyroid nodules that were surgically confirmed papillary thyroid cancers between January 2012 and June 2013. BRAF mutation was positive in 48 nodules and negative in 48 nodules. For analysis, ROIs from the nodules were demarcated manually on both longitudinal and transverse sonographic images. We extracted a total of 86 radiomics features derived from histogram parameters, gray-level co-occurrence matrix, intensity size zone matrix, and shape features. These features were used to build 3 different classifier models, including logistic regression, support vector machine, and random forest using 5-fold cross-validation. The performance including accuracy, sensitivity, specificity, positive predictive value, negative predictive value, and area under the receiver operating characteristic curve, of the different models was evaluated.
\end{abstract}

RESULTS: The incidence of high-suspicion nodules diagnosed on ultrasound was higher in the BRAF mutation-positive group than in the mutation-negative group $(P=.004)$. The radiomics approach demonstrated that all classification models showed moderate performance for predicting the presence of BRAF mutation in papillary thyroid cancers with an area under the curve value of 0.651 , accuracy of $64.3 \%$, sensitivity of $66.8 \%$, and specificity of $61.8 \%$, on average, for the 3 models.

CONCLUSIONS: Radiomics study using thyroid sonography is limited in predicting the BRAF mutation status of papillary thyroid carcinoma. Further studies will be needed to validate our results using various diagnostic methods.

ABBREVIATIONS: AUC = area under the receiver operating characteristic curve; GLCM = gray-level co-occurrence matrix; ISZM = intensity size zone matrix; K-TIRADS = Korean Thyroid Imaging Reporting and Data System; mRMR = minimum redundancy maximum relevance; PTC = papillary thyroid carcinoma; $\mathrm{US}=$ ultrasound

P apillary thyroid carcinoma (PTC) is the most common type of thyroid malignancy and accounts for the rapidly increasing incidence of thyroid cancer worldwide. ${ }^{1,2}$ The B-Raf proto-oncogene, serine/threonine kinase (BRAF) mutation plays a central role

Received August 9, 2019; accepted after revision December 17

From the Department of Radiology (M.-r.K., J.H.S., S.Y.H., K.W.P.), Samsung Medical Center, Sungkyunkwan University School of Medicine, Seoul, Republic of Korea;

Department of Radiology (M.-r.K.), Kangbuk Samsung Hospital, Sungkyunkwan

University School of Medicine, Seoul, Republic of Korea; Center for Neuroscience Imaging Research/School of Electronic and Electrical Engineering (H.P.),

Sungkyunkwan University, Suwon, Korea; and Center for Neuroscience Imaging

Research/Department of Electronic and Computer Engineering (H.C.),

Sungkyunkwan University, Suwon, Korea.

Jung Hee Shin and Hyunjin Park contributed equally to this study

This work was supported by the Institute for Basic Science (grant No. IBS-R015-D1), the National Research Foundation of Korea (grant No. NRF-2019R1H1A2079721), the Ministry of Science and ICT of Korea under the Information Technology Research Center support program (grant No. IITP-2019-2018-0-01798) supervised by the Institute for Information and Communication Technology Promotion, and the Institute for Information and Communication Technology Promotion grant funded by the Korean government under the AI Graduate School Support Program (grant No. 2019-0-00421). in the pathogenesis of PTC, promoting carcinogenesis through the action of the mitogen-activated protein kinase pathway. The frequency of the BRAF mutation in PTC has been reported to range from $29 \%$ to $83 \%$ and is known to be the most common genetic alteration in PTC. ${ }^{5,6}$ Many studies have reported that the BRAF mutation is associated with poor clinicopathologic outcomes, such as a high incidence of advanced clinical stage, extrathyroidal extension, and increased recurrence. ${ }^{6-9}$ These results suggest that preoperative knowledge of the BRAF mutation status can be helpful in categorizing patients as

\footnotetext{
Please address correspondence to Hyunjin Park, PhD, Center for Neuroscience Imaging Research/School of Electronic and Electrical Engineering, Sungkyunkwan University, Suwon, 16419, Korea; e-mail: hyunjinp@skku.edu

- Indicates open access to non-subscribers at www.ajnr.org

三 Indicates article with supplemental on-line tables.

Indicates article with supplemental on-line photo

http://dx.doi.org/10.3174/ajnr.A6505
} 
high risk and planning an appropriate treatment strategy. According to 2015 American Thyroid Association guidelines, active surveillance of PTC has emerged as a safe alternative to surgical intervention in low-risk patient with PTCs. ${ }^{10}$ In this era, preoperative knowledge of the $B R A F$ mutation status can be one of preoperative modulators for planning an appropriate treatment strategy, such as the determination of an early surgical intervention.

Several studies have investigated whether gray-scale ultrasound (US) findings could predict the presence of the BRAF mutation in PTC and have reported controversial results. Kabaker et $\mathrm{al}^{11}$ reported that most of the suspicious US findings, including a taller-than-wide shape, ill-defined margin, hypoechogenicity, calcifications, and absent halo were associated with $B R A F$ mutation positivity, and Hahn et $\mathrm{al}^{12}$ reported that hypoechogenicity and nonparallel orientation were associated with $B R A F$ mutation positivity. Conversely, other studies have found no close correlation between suspicious US features and the BRAF mutation., 93,14

With these various results, visual interpretation of US images has limitations, including a high dependency on the radiologist's experience and interobserver variation. In addition, substantial objective information from the image may not be evaluated through visual interpretation. Radiomics, which automatically extracts innumerable high-dimensional features from images, has recently emerged and shows promising results for decision support. ${ }^{15}$ Previous studies have reported that histograms and texture analyses of US are useful for differentiating benign and malignant thyroid nodules. ${ }^{16-21}$ To our knowledge, there have been no published studies aimed at identifying the presence of $B R A F$ mutation using radiomics features of US.

Therefore, the purpose of this study was to evaluate whether radiomics study of gray-scale US could predict the presence or absence of BRAF mutation in PTC.

\section{MATERIALS AND METHODS \\ Patient Selection}

The institutional review board of our institution (Samsung Medical Center) approved this retrospective study. We retrospectively reviewed our institutional data base to identify patients with surgically confirmed PTC who underwent preoperative thyroid US and successful DNA sequencing for BRAF mutations between January 2012 and June 2013. The exclusion criteria were as follows: 1) nodule diameter of $<10 \mathrm{~mm}$ in small nodules because the ROI method has lower accuracy and current guidelines do not recommend fine-needle aspiration for nodules with a diameter of $<10 \mathrm{~mm} ;{ }^{22,23}$ 2) lack of precise correlation between pathology, the BRAF mutation study, and US findings in patients with multiple nodules; and 3) both transverse and longitudinal US images not being available. Finally, this study included a total of 96 PTCs from 96 patients (mean age, $44.9 \pm 13.2$ years; range, $19-77$ years). The final surgical diagnoses and BRAF mutation results of the thyroid nodules were analyzed.

\section{US Examinations and Image Evaluation}

All patients underwent preoperative thyroid US using an iU22 Vision 2010 machine (Philips Healthcare, Seattle,
Washington) with a commercially available $7-$ to $12-\mathrm{MHz}$ linear-array transducer. All scans were performed by 1 of 7 radiologists with between 2 and 15 years of experience in thyroid US. Longitudinal and transverse images were obtained for each nodule.

One radiologist (M.-r.K.) retrospectively reviewed preoperative US and assessed image features, and another radiologist (J.H.S.) with 15 years of experience in thyroid US supervised this step. According to the Korean Thyroid Imaging Reporting and Data System (K-TIRADS), ${ }^{22}$ all thyroid nodules were evaluated for internal content, echogenicity, shape, orientation, margin, and calcifications. The final category assessment was divided into 5 categories according to the K-TIRADS as follows: category 1, no nodule; category 2, benign nodule; category 3, low-suspicion nodule; category 4, intermediate-suspicion nodule; and category 5 , high-suspicion nodule.

\section{Radiomics Feature Analysis}

The most representative transverse and longitudinal images of each tumor were selected for radiomics feature extraction. An ROI in the thyroid tumor was delineated manually along the border of each tumor on representative US images using MRIcron software (http://www.mricro.com/mricron) by 2 radiologists (M.-r.K. and J.H.S.). The intraclass correlation coefficient was computed to assess the reproducibility of features using 2 sets of ROIs. The first set of ROIs was used for the radiomics analysis.

A total of 86 radiomics features were extracted using open-source radiomics software, Py-Radiomics (https:// www.radiomics.io/pyradiomics.html). ${ }^{24}$ Forty-three features were computed for each technique (transverse and longitudinal images). Features computed from both orientations were considered. The features were grouped into shape (6 features), histogram-based (19 features), intensity size zone matrix (ISZM, 2 features), and gray-level co-occurrence matrix (GLCM, 16 features). The histogram-based features were computed from 64-bin histograms calculated over the intratumoral intensity range. The GLCM features assess textural information and reflect intratumoral heterogeneity using a $2 \mathrm{D}$ histogram with 64 bins. A total of 8 matrices corresponding to eight $2 \mathrm{D}$ directions with an offset of 1 were computed and then averaged to yield a single matrix. The averaged matrix was used to compute the GLCM features. The ISZM features were also related to texture using blobs of similar intensity and differing sizes. We constructed a $32 \times 256$ matrix in which the first dimension was binned intensity and the second dimension was the size of the blobs. Further details regarding the features are given in On-line Tables 1-3.

Due to the lack of external validation data, we applied 5-fold cross-validation to separate our data into training and test sets to reduce overfitting. Models were built using the training set only and tested on a left-out test set. Each model was trained using $80 \%$ of the data $(n=77)$ and later tested on the remaining $20 \%$ of the data $(n=18)$. Feature selection was performed using minimum redundancy maximum relevance (mRMR) from the training set. ${ }^{25}$ The number of 
chosen features of mRMR was set using a grid search between 3 and 11. Within the cross-validation, the optimal number of features was chosen on the basis of the maximum performance in the test set on average for the 3 classifiers (On-line Figure). The selected features were used as input to train 3 different classifier models, including logistic regression, support vector machine using the linear kernel, and random forest with 50 trees. As for tuning the hyperparameters of the support vector machine, we tried different kernels, including linear, quadratic, and radial basis functions for the support vector machine, and linear kernel worked the best. The random forest classifier has feature-selection capabilities. However, the other 2 models, logistic regression and support vector machine, do not have such capabilities. We adopted an external feature-selection procedure (ie, mRMR) so that all 3 models were subjected to the same feature-selection procedure. The trained classifiers were further tested on a left-out test fold. Because we adopted 5-fold cross-validation, we repeated the procedures of feature selection, model training, and testing steps 5 times, each time leaving out a different test fold. The performance of the classifier models was assessed on the basis of accuracy, sensitivity, specificity, positive predictive value, negative predictive value, and area under the receiver operating characteristic curve (AUC).

\section{Statistical Analysis}

To compare BRAF mutation-positive and -negative PTCs, we analyzed categoric variables using a $\chi^{2}$ or Fisher exact test, while continuous variables were analyzed using a Student $t$ test. A $P$ value $<.05$ was considered statistically significant.
Table 1: Clinical and tumor characteristics of study population

\begin{tabular}{lccc}
\hline & BRAF $(+)$ PTC $(\boldsymbol{n}=\mathbf{4 8})$ & BRAF $(-)$ PTC $(\boldsymbol{n}=\mathbf{4 8})$ & P Value \\
\hline Age (mean) (yr) & $46.9 \pm 13.8$ & $42.8 \pm 12.4$ & .13 \\
Sex & & & .27 \\
$\quad$ M & $11(22.9)$ & $7(14.6)$ & \\
$\quad$ F & $37(77.1)$ & $41(85.4)$ & \\
Tumor size (mean) (cm) & $1.59 \pm 0.57$ & $1.87 \pm 1.05$ & .12 \\
Central lymph node metastasis & $16(33.3)$ & & .15 \\
$\quad$ No & $32(66.7)$ & $24(50.0)$ & \\
$\quad$ Yes & & $24(50.0)$ & \\
Lateral lymph node metastasis & $38(79.2)$ & $38(79.2)$ & \\
$\quad$ No & $10(20.8)$ & $10(20.8)$ & \\
$\quad$ Yes & & & \\
\hline
\end{tabular}

Table 2: Relationship between BRAF mutation and US imaging characteristics by visual assessment of papillary thyroid carcinomas

\begin{tabular}{|c|c|c|c|}
\hline & BRAF (+) PTC $(n=48)$ & BRAF $(-)$ PTC $(n=48)$ & $P$ Value \\
\hline Internal content & & & .16 \\
\hline Solid & $46(95.8)$ & $41(85.4)$ & \\
\hline Predominantly solid & $2(4.2)$ & $7(14.6)$ & \\
\hline Echogenicity & & & .012 \\
\hline Markedly hypoechoic & $20(41.7)$ & $8(16.7)$ & \\
\hline Hypoechoic & $23(47.9)$ & $26(54.2)$ & \\
\hline Isoechoic & $5(10.4)$ & $13(27.1)$ & \\
\hline Hyperechoic & $0(0.0)^{\prime}$ & $1(2.1)^{\prime}$ & \\
\hline Shape & & & .08 \\
\hline Irregular & $12(25.0)$ & $20(41.7)$ & \\
\hline Round to oval & $36(75.0)$ & $28(58.3)$ & \\
\hline Orientation & & & .007 \\
\hline Nonparallel & $26(54.2)$ & $13(27.1)$ & \\
\hline Parallel & $22(45.8)$ & 35 (72.9) & \\
\hline Margin & & & .66 \\
\hline Spiculated/microlobulated & $35(72.9)$ & $31(64.6)$ & \\
\hline Ill-defined & $4(8.3)$ & $6(12.5)$ & \\
\hline Smooth & $9(18.8)$ & $11(22.9)$ & \\
\hline Calcification & & & .33 \\
\hline No & $18(37.5)$ & $16(33.3)$ & \\
\hline Microcalcification & 19 (39.6) & $22(45.8)$ & \\
\hline Macrocalcification & $6(12.5)$ & $9(18.8)$ & \\
\hline Rim calcification & $5(10.4)$ & $1(2.1)^{\prime}$ & \\
\hline Final K-TIRADS category & & & .004 \\
\hline 3 (Low suspicion) & $1(2.1)$ & $3(6.3)$ & \\
\hline 4 (Intermediate suspicion) & 9 (18.8) & $22(45.8)$ & \\
\hline 5 (High suspicion) & $38(79.2)$ & $23(47.9)$ & \\
\hline
\end{tabular}

\section{RESULTS}

The clinical, pathologic, and imaging findings of 96 patients are shown in Tables 1 and 2. Among the total of 96 patients, 78 were women (mean age, $45.1 \pm 13.9$ years; range, 19-77 years) and 18 were men (mean age, $43.9 \pm$ 10.2 years; range, $21-62$ years).

The 96 nodules consisted of 48 $B R A F$ mutation-positive PTCs and 48 $B R A F$ mutation-negative PTCs. Clinical characteristics, including patient age and sex, were not significantly different. The mean tumor size was $1.73 \pm 0.85 \mathrm{~cm}$ (range, $1-6 \mathrm{~cm}$ ). The mean tumor size was not significantly different between the 2 groups (BRAF mutation-positive group: $1.59 \pm 0.57 \mathrm{~cm}$; $B R A F$ mutation-negative group: $1.87 \pm 1.05 \mathrm{~cm}$, $P=.12)$. Central and lateral lymph node metastases were not significantly different $(P=.15$ and $P=1.00$, respectively). Among US characteristics, echogenicity was significantly different between the 2 groups $(P=.012)$. BRAF mutation positive groups significantly showed nonparallel orientation $(P=.007)$ (Table 2). The incidence of K-TIRADS category 5 (high suspicion) was higher than that of K-TIRADS 3 (low suspicion) or 4 (intermediate suspicion) in the BRAF mutation-positive group $(P=.004)$. The intraclass correlation coefficient of 86 features was a mean of $0.89 \pm$ of 0.09 as shown in On-line Table 4.

We adopted 5-fold cross-validation; thus, the selected features varied from fold to fold. There were 2 features that were selected $>3$ times: the mean 
(histogram) of the longitudinal image and informational measure of correlation (GLCM) of the longitudinal image. We reported the mean values of the performance metric along with confidence intervals over the 5 folds in On-line Table 5. The averages for the 3 classifier models were as follows: accuracy, 64.3\% (range, 63.68\%-64.68\%); sensitivity, 66.8\% (range, 64.67\%-70.89\%); and specificity, $61.8 \%$ (range, 58.22\%-64.67\%). The receiver operating characteristic of the 3 models yielded a relatively low AUC of 0.65 on average (range, 0.6446-0.6562) (Figure).

We also performed a different feature-selection approach (ie, Pearson correlation-based feature selection) to see if it led to better performance. We computed the correlation between all possible pairs of features, and if the correlation exceeded 0.5, we kept the feature that had a higher correlation with the mutation status for a given pair of features. After feature selection, 3 models were trained and tested. The results of 3 classifiers using a simple Pearson correlation-based feature selection are given in On-line Table 6. Results showed that training performance was better than those using the mRMR feature selection, but test performance was worse, which implied that models were overfitting. The averages for the 3 classifier models were as follows: accuracy, $58.6 \%$ (range, 56.16\%-60.42\%); sensitivity, 63.9\% (range, 60.44\%-66.89\%); and specificity, 53.8\% (range, 52.22\%-56.67\%). The receiver operating characteristic of the 3 models yielded a relatively low AUC of 0.61 on average. The test performance in terms of AUC showed the lower bound of the confidence interval as 0.52 , slightly above the chance level (ie, 0.50 ).

\section{DISCUSSION}

Since the introduction of radiomics, many previous studies have tried to investigate the relationship between image characteristics and genetic mutations in various malignancies, including lung, colon, brain, and breast cancers. ${ }^{26-29}$ They proposed a CT- or MR imaging-based radiomics model to detect gene mutation status as a noninvasive method. These models were useful to predict the presence of gene mutations in malignancies.

To our knowledge, this is the first study to apply radiomics in the estimation of BRAF mutation in patients with PTC. We evaluated the ability of radiomics, using various machine learning approaches, to help predict the presence of the $B R A F$ mutation in patients with PTC. In our study, BRAF-mutated PTCs tended to show nonparallel orientation and marked hypoechogenicity, similar to findings in some previous studies. ${ }^{12,13}$ Although visual assessment of thyroid nodules suggested that high-suspicion findings on US were significantly more frequent in BRAF-mutated PTCs, radiomics demonstrated that all classification models failed to show excellent performance for predicting the presence of $B R A F$ mutation in PTCs.

Radiomics is usually performed using tomographic images, including CT, MR imaging, or PET images because these modalities can acquire $3 \mathrm{D}$ volume data and data acquisition can be standardized by setting scan parameters of the machines so that they are identical. ${ }^{15}$ US has several limitations in quantitative analysis in contrast to tomographic images: Only 2D data can be acquired through this technique along with lack of representative features due to a limited amount of image data, operator dependency, and dependency on US machines. ${ }^{30}$ These factors may have

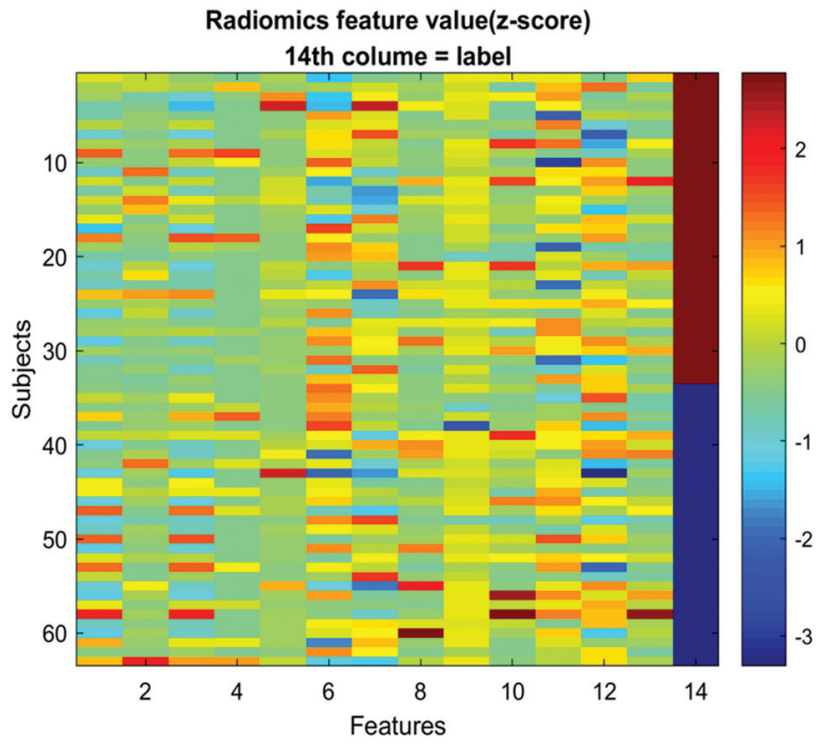

FIGURE. Heatmap showing the correlation of radiomics features with $B R A F$ mutation prediction after feature selection using $\mathrm{mRMR}$. Red indicates higher correlation relative to blue.

affected our results. However, US is the most widely used standard imaging tool in thyroid pathology and is very helpful in discriminating between malignant and benign thyroid nodules. Until now, a number of studies have been published that have reported that quantitative features extracted from US images have favorable results. ${ }^{16-21}$ Further studies with a larger amount of data will be necessary.

In our study, 3 different classifiers were applied to demonstrate the effectiveness of the chosen features. A simple model, such as logistic regression, has few parameters and is interpretable; conversely, complex models, such as support vector machine and random forest, are difficult to interpret and have many parameters. No superiority among the classifiers was noted in this study, and the difference among the AUCs of the 3 classifiers was very small; this finding indicates that choosing any classifier did not affect the overall performance. One possible reason for this result could be that the selected features were not very effective; thus, the results remained comparable regardless of the simple or complex classifier model.

In many studies using machine learning, the performance of the unseen test set tends to be lower than that of the training set because data overfitting might occur in the training set. In particular, using too many trees in the random forest classifier might inflate the performance measures in the training set. ${ }^{31} \mathrm{We}$ conducted a Pearson correlation, one of the other ways to perform feature selection. Results showed that training performance increased, but test performance decreased; these findings imply that the selected features overfitted the training data.

Two features, mean (histogram-based feature) and informational measures of correlation (texture feature) of the longitudinal image, were selected $>3$ times. Thus, they were important features to explain the $B R A F$ mutation. There was a relatively small overlap between the 2 features when we computed a Pearson correlation ( $r=0.15$ with $P=.14$ ). The mean value reflects echogenicity of the ROI, which is compatible with our visual assessment 
of US images. ${ }^{16}$ The informational measure of correlation is related to the heterogeneity of the ROI and thus could have a potential correlation with pathology. BRAF genetic alterations coexist in thyroid tumors in which some cells provide a basis for mutation and others do not have mutations, forming intratumor heterogeneity. Intratumor heterogeneity may foster tumor evolution and adaptation. ${ }^{32,33}$

Our study had several limitations. First, this was a retrospective study from a single institution, which introduces the possibility of selection bias. Additionally, the small datasets in our study made it difficult to achieve reliable results. With added samples, applying deep learning approaches combined with electronic medical records might be possible; this process might improve the overall performance. Second, although preoperative US was performed with the same US machine set with similar parameters to avoid equipment-based variability, this feature and patient-related factors may still have influenced the pixel intensity of US images. ${ }^{30}$ Third, we focused on predicting the BRAF status of patients with papillary thyroid cancer. Our main goal was not to contrast healthy controls and patients with papillary thyroid cancer. Still, including healthy controls would lead to less positive bias. Last, the lack of external validation data is also a limitation of this study. Our results from this study need to be further validated in a larger dataset to better assess their potential clinical use.

\section{CONCLUSIONS}

Our preliminary study shows that radiomics study of thyroid US was limited in predicting BRAF mutation in PTC.

Disclosures: Hyunjin Park—RELATED: Grant: Institute for Basic Science, National Research Foundation of Korea, Ministry of Science and information and communication technology, Institute for Information and Communication Technology Promotion.* *Money paid to the institution.

\section{REFERENCES}

1. Davies L, Welch HG. Increasing incidence of thyroid cancer in the United States, 1973-2002. JAMA 2006;295:2164-67 CrossRef Medline

2. Leenhardt L, Grosclaude P, Cherie-Challine L; Thyroid Cancer Committee. Increased incidence of thyroid carcinoma in France: a true epidemic or thyroid nodule management effects? Report from the French Thyroid Cancer Committee. Thyroid 2004;14:1056-60 CrossRef Medline

3. Xing M. BRAF mutation in thyroid cancer. Endocr Relat Cancer 2005;12:245-62 CrossRef Medline

4. Melck AL, Yip L, Carty SE. The utility of BRAF testing in the management of papillary thyroid cancer. Oncologist 2010;15:1285-93 CrossRef Medline

5. Kim KH, Kang DW, Kim SH, et al. Mutations of the BRAF gene in papillary thyroid carcinoma in a Korean population. Yonsei Med J 2004;45:818-21 CrossRef Medline

6. Lee JH, Lee ES, Kim YS. Clinicopathologic significance of BRAF V600E mutation in papillary carcinomas of the thyroid: a metaanalysis. Cancer 2007;110:38-46 CrossRef Medline

7. Xing M, Westra WH, Tufano RP, et al. BRAF mutation predicts a poorer clinical prognosis for papillary thyroid cancer. J Clin Endocrinol Metab 2005;90:6373-79 CrossRef Medline

8. Nikiforova MN, Kimura ET, Gandhi M, et al. BRAF mutations in thyroid tumors are restricted to papillary carcinomas and anaplastic or poorly differentiated carcinomas arising from papillary carcinomas. J Clin Endocrinol Metab 2003;88:5399-404 CrossRef Medline
9. Kwak JY, Kim EK, Chung WY, et al. Association of BRAFV600E mutation with poor clinical prognostic factors and US features in Korean patients with papillary thyroid microcarcinoma. Radiology 2009;253:854-60 CrossRef Medline

10. Haugen BR, Alexander EK, Bible KC, et al. 2015 American Thyroid Association Management Guidelines for Adult Patients with Thyroid Nodules and Differentiated Thyroid Cancer: The American Thyroid Association Guidelines Task Force on Thyroid Nodules and Differentiated Thyroid Cancer. Thyroid 2016;26:1-133 CrossRef Medline

11. Kabaker AS, Tublin ME, Nikiforov YE, et al. Suspicious ultrasound characteristics predict BRAF V600E-positive papillary thyroid carcinoma. Thyroid 2012;22:585-89 CrossRef Medline

12. Hahn SY, Kim TH, Ki CS, et al. Ultrasound and clinicopathological features of papillary thyroid carcinomas with BRAF and TERT promoter mutations. Oncotarget 2017;8:108946-57 CrossRef Medline

13. Hwang J, Shin JH, Han BK, et al. Papillary thyroid carcinoma with BRAFV600E mutation: sonographic prediction. AJR Am J Roentgenol 2010;194:W425-30 CrossRef Medline

14. Li Q, Yuan J, Wang Y, et al. Association between the BRAF V600E mutation and ultrasound features of the thyroid in thyroid papillary carcinoma. Oncol Lett 2017;14:1439-44 CrossRef Medline

15. Gillies RJ, Kinahan PE, Hricak H. Radiomics: images are more than pictures, they are data. Radiology 2016;278:563-77 CrossRef Medline

16. Kwon MR, Shin JH, Hahn SY, et al. Histogram analysis of greyscale sonograms to differentiate between the subtypes of follicular variant of papillary thyroid cancer. Clin Radiol 2018;73:591e1-e7 Medline Medline

17. Song G, Xue F, Zhang C. A model using texture features to differentiate the nature of thyroid nodules on sonography. J Ultrasound Med 2015;34:1753-60 CrossRef Medline

18. Chen SJ, Chang CY, Chang KY, et al. Classification of the thyroid nodules based on characteristic sonographic textural feature and correlated histopathology using hierarchical support vector machines. Ultrasound Med Biol 2010;36:2018-26 CrossRef Medline

19. Nam SJ, Yoo J, Lee HS, et al. Quantitative evaluation for differentiating malignant and benign thyroid nodules using histogram analysis of grayscale sonograms. J Ultrasound Med 2016;35:775-82 CrossRef Medline

20. Grani G, D’Alessandri M, Carbotta G, et al. Grey-scale analysis improves the ultrasonographic evaluation of thyroid nodules. Medicine (Baltimore) 2015;94:e1129 CrossRef Medline

21. Bhatia KS, Lam AC, Pang SW, et al. Feasibility study of texture analysis using ultrasound shear wave elastography to predict malignancy in thyroid nodules. Ultrasound Med Biol 2016;42:1671-80 CrossRef Medline

22. Shin JH, Baek JH, Chung J, et al; Korean Society of Thyroid Radiology (KSThR) and Korean Society of Radiology. Ultrasonography diagnosis and imaging-based management of thyroid nodules: revised Korean Society of Thyroid Radiology consensus statement and recommendations. Korean J Radiol 2016;17:370-95 CrossRef Medline

23. Tessler FN, Middleton WD, Grant EG, et al. ACR Thyroid Imaging, Reporting and Data System (TI-RADS): White Paper of the ACR TIRADS Committee. J Am Coll Radiol 2017;14:587-95 CrossRef Medline

24. van Griethuysen JJ, Fedorov A, Parmar C, et al. Computational radiomics system to decode the radiographic phenotype. Cancer Res 2017;77:e104-07 CrossRef Medline

25. Peng H, Long F, Ding C. Feature selection based on mutual information: criteria of max-dependency, max-relevance, and min-redundancy. IEEE Trans Pattern Anal Mach Intell 2005;27:1226-23 CrossRef Medline

26. Yang L, Dong D, Fang M, et al. Can CT-based radiomics signature predict KRAS/NRAS/BRAF mutations in colorectal cancer? Eur Radio 2018;28:2058-67 CrossRef Medline

27. Liu Y, Kim J, Balagurunathan Y, et al. Radiomic features are associated with EGFR mutation status in lung adenocarcinomas. Clin Lung Cancer 2016;17:441-48e6 CrossRef Medline 
28. Li $\mathrm{H}, \mathrm{Zhu} \mathrm{Y}$, Burnside ES, et al. MR imaging radiomics signatures for predicting the risk of breast cancer recurrence as given by research versions of MammaPrint, Oncotype DX, and PAM50 gene assays. Radiology 2016;281:382-91 CrossRef Medline

29. Chen X, Tong Y, Shi Z, et al. Noninvasive molecular diagnosis of craniopharyngioma with MRI-based radiomics approach. $B M C$ Neurol 2019;19:6 CrossRef Medline

30. Lee SE, Han K, Kwak JY, et al. Radiomics of US texture features in differential diagnosis between triple-negative breast cancer and fibroadenoma. Sci Rep 2018;8:13546 CrossRef Medline
31. Breiman L. Random forests. Machine Learning 2001;45:5-32 CrossRef

32. Guerra A, Sapio MR, Marotta V, et al. The primary occurrence of BRAF(V600E) is a rare clonal event in papillary thyroid carcinoma. J Clin Endocrinol Metab 2012;97:517-24 CrossRef Medline

33. Guerra A, Fugazzola L, Marotta V, et al. A high percentage of BRAFV600E alleles in papillary thyroid carcinoma predicts a poorer outcome. J Clin Endocrinol Metab 2012;97:2333-40 CrossRef Medline 